\title{
WEIGHT-BAND DOSING TABLES: SIMPLIFYING PAEDIATRIC ART
}

\author{
James J C Nuttall, $M B C h B, D T M \& H$ \\ Paediatric Infectious Diseases Unit, Red Cross Children's Hospital and University of Cape Town
}

\begin{abstract}
One of the obstacles to scaling up paediatric antiretroviral therapy (ART) coverage in resource-limited settings is the relative complexity of paediatric dosing. There is a need to simplify ART in order to facilitate treatment initiation and ongoing management of infants and children by health care providers, as well as to support adherence in the home. This article reviews the development of weight-band dosing tables as a strategy for simplifying the delivery of paediatric ART.
\end{abstract}

In 2007, only $8 \%$ of the estimated 1800000 children (0 - 14 years) living with HIV in sub-Saharan Africa were receiving antiretroviral therapy (ART). Coverage will need to be expanded greatly if the goal of providing ART to $80 \%$ of children in need by 2010 is to be met. ${ }^{1}$ Moreover, recent evidence highlights early initiation of ART as particularly critical for infants with HIV. ${ }^{2}$ Clinical guidelines issued by the World Health Organization (WHO) now recommend immediate initiation of ART for all HIV-infected infants. ${ }^{3}$

Paediatric ART management involves a complex process of interactions between patients, families, health care providers and the antiretrovirals (ARVs) themselves. Barriers to the delivery of effective treatment occur both within the health care system and in the home. These include delayed diagnosis, limited availability of health care providers trained in paediatric ART, few available paediatric ARV formulations, complicated regimens and dosing schedules, and poor palatability of some ARVs. Difficulties in the home include overcrowding, difficult work schedules of the parents and the stresses associated with parental disclosure in the home. Unlike adults, children require changes in antiretroviral dose as they grow and become older, and rely upon adult caregivers to administer medicines. ${ }^{4}$

Children have traditionally been dosed according to body surface area (BSA) (e.g. zidovudine (AZT), didanosine (ddl), lopinavir/ritonavir (LPV/r)), weight $(\mathrm{mg} / \mathrm{kg})$ (e.g. stavudine (d4T), lamivudine (3TC), abacavir (ABC), nevirapine (NVP)) or dose per weight band (efavirenz (EFV)). Manufacturers' recommendations for some ARV drugs (e.g. LPV/r) include both BSA and weight-based dosing methods. ${ }^{5}$ The calculation of BSA generally requires accurate measurement of the child's weight and length or height (ideally with a stadiometer or measuring box), and a normogram or mathematical formula (e.g. Mosteller formula). ${ }^{6}$ For both BSA and $\mathrm{mg} / \mathrm{kg}$ weight-based dosing approaches, the calculated dose of each ARV drug must be rounded up or down as a 'best-fit' dosage according to which solid or liquid formulations of the ARV drug are available. This may lead to confusion and uncertainty on the part of the prescriber.

In resource-limited settings (RLS), primary care doctors and nurses rather than paediatricians are responsible for the majority of paediatric ART initiation and follow-up. Lack of accurate measuring equipment and the relative complexity of BSA dosing may inhibit initiation of ART in infants and young children or mean that inappropriate doses are given. While the most 'accurate' dosing may be obtained with the use of liquid formulations, large volumes of solutions (which may require refrigeration, e.g. d4T solution) may be challenging for caregivers to administer to young children, particularly if palatability is poor (e.g. LPV/r).

ART simplification strategies are required to help health care providers manage ART in children, and to help caregivers and children adhere to therapy. Interventions include the use of adult or preferably paediatric fixed-dose combinations (FDCs), selection of doses based on weight band rather than individual $\mathrm{mg} / \mathrm{kg}$ or BSA doses, prescription of pills or capsules rather than liquids, and identification of reliable oncedaily regimens.

MOVING FROM BSA AND WEIGHT-BASED DOSING APPROACHES TO WEIGHT-BAND DOSAGE TABLES

Weight-band dosage tables assist health care providers by assigning a fixed dose of medication for a particular weight range (e.g. $1.5 \mathrm{ml}$ of $\mathrm{LPV} / \mathrm{r}$ solution for children weighing $4-9.9 \mathrm{~kg}$, or half a $150 \mathrm{mg}$ 3TC tablet for children weighing $14-19.9 \mathrm{~kg}$ ). In large public sector 
treatment programmes, tables can reduce the time and risk of dosing errors involved in calculating multiple ARV doses by weight or BSA. They can also facilitate easier checking of doses against weight gain by clinicians, nurses, pharmacists or adherence counsellors. Under-dosing of ARVs, due in part to a lack of regular dose adjustments for ongoing growth, has been described in a large cohort of children in the UK and Ireland. ${ }^{7}$ A study in Thailand found that 17 of 18 doctors using a standardised drug dosage table avoided miscalculations and reported more confidence with prescriptions. $^{8}$

In 2006, the WHO published simplified weight-band dosing tables on all ARV drugs for which there were available paediatric indications, formulations or sufficient information and evidence to provide guidance on prescribing and doses. ${ }^{9}$ Decisions about dosing were based upon manufacturers' information, ARV formulation choices, available data from clinical studies and expert paediatric pharmacology consultation. What was considered to be the 'optimal' dose for a particular weight band, given the limitations imposed by currently available drug formulations, was selected. Weightband doses were determined by using BSA values calculated from median height-for-weight from international growth charts using the following formula: $\mathrm{BSA}=$ square root [[weight $(\mathrm{kg}) \times$ height $(\mathrm{cm})] / 3600]$.

The dosing tables are directed at RLS and are based on the following principles:

1. It is preferable to use one formulation or fixed combination of any given drug(s).

2. Syringes or other standardised devices of various sizes should be available to support accurate dosing of liquid formulations.

3. Large volumes of liquid or syrup formulations should be avoided where possible.

4. In general, children should be switched to available solid formulations as soon as possible or as soon as they are tolerated.

5. If liquids or syrups are difficult because of storage, large volumes required or palatability, solid dosage formulations are preferable.

6. If solid formulations of first-line and second-line drugs developed for children are unavailable, solid formulations currently used for adults can be used.

7. Many tablets, but not all, may be divided in half but not beyond as drug content cannot be guaranteed. Scored tablets are more easily split. Some tablets cannot be split, and the WHO recommends that where possible tablet splitting be done in the dispensing pharmacy using appropriate tablet cutters.

8. Some adult FDCs may result in under-dosing of individual components in children. This is of con- cern, particularly with drugs such as NNRTIs and 3TC where there is a low threshold for resistance. NVP requires a 'lead-in' dosage. During the first 2 weeks, therefore rather use individual components of the regimen.

9. Different dosing between a.m. and p.m. should be avoided where possible. However, in order to keep all regimens to no more than twice daily, there are instances where different quantities of solid dosage forms can be administered a.m. as opposed to p.m.

10. The doses in the tables are presented in weight bands, accepting that some deviation from target dosing will occur.

11. Children have to be weighed at each clinic visit, and dose changes are required as children grow and/or gain weight.

12. When capsules are opened or tablets dissolved or crushed and added to food or liquid, it is important that the entire volume/amount of vehicle be taken to ensure administration of the full dose.

The dosing tables are based on standardised weight bands starting from $5 \mathrm{~kg}$ body weight for the individual ARV drugs (excluding EFV, which starts from $10 \mathrm{~kg}$ ) and $10-14 \mathrm{~kg}$ for the fixed-dose combinations (AZT + 3TC, $d 4 T+3 T C, A Z T+3 T C+A B C, d 4 T+3 T C+N V P)$. The weight bands are in $1 \mathrm{~kg}$ divisions from 5 to $11 \mathrm{~kg}$, and 2 - $5 \mathrm{~kg}$ divisions from 12 to 35 or $40 \mathrm{~kg}$.

\section{ADAPTING WEIGHT-BAND DOSING TABLES FOR DIFFERENT SETTINGS}

Dosing tables may be adapted according to the specific drug formulations available to a regional or national treatment programme. For example, dual (d4T $+3 \mathrm{TC}$ ) or triple (d4T or AZT + 3TC + NVP) FDCs play an important role in paediatric ART in many countries (e.g. Thailand and many African countries), but are not widely used in South Africa. Satisfactory early clinical and immunological outcomes have been described following the use of fractions of generic adult FDCs in children dosed according to a weight-band table method in Thailand. ${ }^{10}$

In another project, a visual dosing aid (VDA) incorporating coloured dosing bands for five first-line ARV drugs was developed to assist clinicians in prescribing paediatric ART consisting of syrups, generic adult tablets or a combination. It compared well with generic paediatric FDC tablets and could help facilitate paediatric ART roll-out in RLS. ${ }^{11}$

The South African national guidelines for the management of HIV-infected children (2005) incorporate a weight-band dosing chart ${ }^{12,13}$ developed by the Centers for Disease Control and Prevention (CDC) and a number of international paediatric AIDS programmes 
prior to the more widespread availability of FDCs. BSA dosing recommendations were converted to weightband doses using approximations of weight-for-age and height-for-age derived from standardised growth charts of girls from birth to 36 months and 20 years in the USA (National Center for Health Statistics). Dose for each weight band was based on the practicality of the available dosage forms for each drug, and practical storage and dosing instructions were included with the chart. The chart lacks dose recommendations for children weighing less than $5-7 \mathrm{~kg}$ and for HIV-TB co-infection.

The Western Cape Antiretroviral Drug Dosing Chart for Children (2007), based on the 2006 WHO recommendations, was developed by the author and colleagues as a pilot project for the HIV Directorate of the Western Cape (South Africa) provincial health department. The chart was directed at both clinicians and pharmacists involved in prescribing and dispensing ART for children, and it was successfully piloted at a number of HIV clinics before being distributed across the province. It was produced as a laminated A4 colour copy. Standard first- and second-line ART regimens (including regimens compatible with rifampicin-based TB treatment) as well as general comments relating to storage, administration and common drug interactions and sideeffects of the individual ARV drugs were printed on the reverse side. Only ARV formulations available at public sector treatment facilities were included (no FDCs were available), and there was an emphasis on the early introduction of solid formulations where possible. Fractions of tablets (not less than half a tablet) were only incorporated for scored tablets. The target dose in $\mathrm{mg} /$ $\mathrm{kg}$ or $\mathrm{mg} / \mathrm{m}^{2}$ for each drug as well as the formula for calculating BSA was included to facilitate comparison of dosing methods when necessary.

The following local practices were incorporated:

1. The off-label 'opened capsule' d4T dosing method whereby a d4T capsule is opened and the powder contents dispersed in a standardised volume of water and the required dose drawn up with a syringe and administered to the child (e.g. to administer a standardised d4T dose of $10 \mathrm{mg}$ for the weight bands $7-9.9 \mathrm{~kg}$, a $20 \mathrm{mg}$ d4T capsule is opened and dispersed into $5 \mathrm{ml}$ of water and $2.5 \mathrm{ml}$ is withdrawn with a syringe and administered and the remaining $2.5 \mathrm{ml}$ is discarded). The rationale for this method is to minimise the use of stavudine solution ( $1 \mathrm{mg} / \mathrm{ml}$ when reconstituted), which is expensive, requires refrigeration, and results in relatively large medication volumes for administration to young infants at the usual dosing schedule ( $1 \mathrm{mg} / \mathrm{kg} /$ dose). There are now data based on high-performance liquid chromatography analysis of active drug concentration in dispersed capsule solutions, supporting the accuracy of this method for certain brands of d4T capsules. ${ }^{14}$

2. Standardised doses of ritonavir used for pharmacological boosting of LPV/r (in order to achieve a ratio of $1: 1$ ) in children receiving concurrent $L P V / r$ and rifampicin-based TB treatment. ${ }^{15}$

3. Colour coding of ARVs on the chart corresponding to colour coding methods used in the pharmacy for medication containers and syringes to assist parents and caregivers with correct dosing.

4. The chart incorporated co-trimoxazole and multivitamin syrup dosing according to weight bands.

5. Since there were no standard weight-band dosing recommendations for infants weighing $<5 \mathrm{~kg}$ in the WHO document (2006), it was recommended that a clinician experienced in ARV prescribing be consulted for such cases.

\section{VALIDATION OF WEIGHT-BAND DOSING TABLES}

There are a number of approaches to validation of weight-band dosing. Direct methods include pharmacokinetic (Pk) studies and therapeutic drug monitoring. Indirect methods include comparison of weight-based with BSA doses, and safety and efficacy studies. Qualitative studies assessing the usefulness of a weightband dosing table to prescribing clinicians and dispensing pharmacists should be undertaken.

Differing growth rates and the prevalence of malnutrition could have a significant impact on the accuracy of weight-band dosing of drugs that are usually dosed by BSA. A study comparing the calculated BSA dose range with WHO weight-band doses for AZT, ddl, NVP and LPV/r using actual heights and weights of 601 children at the time of ARV initiation was undertaken at a tertiary hospital in South Africa in 2007. ${ }^{16}$ The median age was 28 months (interquartile range 13 - 62), 49\% of children weighed $<10 \mathrm{~kg}$, and $59 \%$ and $63 \%$ of children had weight-for-age and height-for-age z-scores $\leq-2$ (moderate to severe underweight or stunting), respectively. Children with body weight $<5 \mathrm{~kg}$ were excluded as weight-based dosing recommendations were unavailable for this category, and children $<6$ months of age were excluded as LPV/r BSA dosing recommendations are different in this age group. The BSA dose ranges used were AZT $180-240 \mathrm{mg} / \mathrm{m}^{2}$, ddl $90-120 \mathrm{mg} / \mathrm{m}^{2}$, NVP $160-200 \mathrm{mg} / \mathrm{m}^{2}$, and $\mathrm{L} / \mathrm{r} 230-300 \mathrm{mg} / \mathrm{m}^{2}$.

Results are presented in Table I. The conclusion of this study was that the 2006 WHO simplified weight banddosing method effectively avoided under-dosing children in relation to existing $B S A$ dose recommendations for AZT, NVP and LPV/r suspensions. However, the authors noted that the risk of over-dosing is greater with weight-band recommendations for existing capsule or tablet formulations of these ARVs. Further studies are recommended for the WHO weight-band dosing method. 


\begin{tabular}{|c|c|c|}
\hline \multirow{2}{*}{$\begin{array}{l}\text { ARV drug, BSA dose range, } \\
\text { formulations assessed }\end{array}$} & \multicolumn{2}{|c|}{$\begin{array}{l}\text { Weight-band dose relative to calculated BSA dose } \\
\text { range ( } N=601 \text { children) (using anthropometric data } \\
\text { at time of starting ART) }\end{array}$} \\
\hline & Under-dosing (\% of children) & Over-dosing (\% of children) \\
\hline \multicolumn{3}{|l|}{ AZT } \\
\hline \multicolumn{3}{|l|}{$180-240 \mathrm{mg} / \mathrm{m}^{2}$} \\
\hline Oral solution $(10 \mathrm{mg} / \mathrm{ml})$ & 2 & 0.5 \\
\hline Capsules (100 mg) & 8.5 & 19 \\
\hline \multicolumn{3}{|l|}{ ddl } \\
\hline \multicolumn{3}{|l|}{$90-120 \mathrm{mg} / \mathrm{m}^{2}$} \\
\hline Tablets $(25,50,100 \mathrm{mg}$, chewable or dispersable in water) & 0 & 87 \\
\hline \multicolumn{3}{|l|}{ NVP } \\
\hline \multicolumn{3}{|l|}{$160-200 \mathrm{mg} / \mathrm{m}^{2}$} \\
\hline Oral suspension $(10 \mathrm{mg} / \mathrm{ml})$ & 0 & 53 \\
\hline Tablets $(200 \mathrm{mg})$ & 0 & 61 \\
\hline \multicolumn{3}{|l|}{ Lopinavir/ritonavir } \\
\hline \multicolumn{3}{|l|}{$230-300 \mathrm{mg} / \mathrm{m}^{2}$} \\
\hline Oral solution $(80 / 20 \mathrm{mg} / \mathrm{ml})$ & 1.2 & 26 \\
\hline Capsules (133.3/33.3 mg soft) & 0 & 38 \\
\hline
\end{tabular}

A VDA to facilitate dosing calculations in response to children's growth and weight during ARV treatment developed by Callens et al." was evaluated using anthropometric data from 55 children from the USA and 324 children from the Democratic Republic of Congo (DRC). In comparison with WHO-recommended dosing, the authors noted a relative dosing difference of $\geq 20 \%$ in $<3 \%$ of children for NVP, AZT and d4T but in $20 \%$ of children for 3TC, over-dosing being more frequent. ${ }^{11}$

A detailed review of Pk studies and clinical outcome, safety and efficacy studies undertaken in children treated with individual or FDC ARV drugs dosed according to weight bands is beyond the scope of this article. There are no reported Pk or clinical studies directly comparing weight band with $\mathrm{mg} / \mathrm{kg}$ or BSA dosing approaches.

\section{CURRENT WEIGHT-BAND TABLES}

In July 2008, the WHO published a revised weightband dosing table for individual ARVs as well as dual and triple FDCs applicable to children $\geq 6$ weeks of age, indicating the number of tablets or millilitres of solution to be administered twice daily by weight band from $3 \mathrm{~kg}$ to $34.9 \mathrm{~kg}$ (Fig. 1). ${ }^{17}$ The table focuses on ARVs used in first-line regimens and was developed by the WHO Paediatric Antiretroviral Working Group using the 2006 WHO treatment recommendations, target doses and weight bands as a benchmark and reviewing currently available published and unpublished data to assess dosing. Ddl is not included in the dosing table. Key supporting references are provided in the document. ${ }^{18}$ See Table II for target doses or dosing ranges.

A WHO generic tool was used to assess and evaluate the expected dose delivered of any product in relation to intended target doses. For all formulations, changes in the number of pills and switches from one formula- tion to another occur at the same weight bands. There was an attempt to avoid dosing any single ARV component below $90 \%$ of the intended delivered dose and not more than 25\% above the intended dose (or dose range for products with an established dose range). For NVP, the aim was to avoid dosing below $100 \%$ of the minimum of the dose range $\left(150 \mathrm{mg} / \mathrm{m}^{2}\right)$. Discrepancies between dose delivered and intended dose were justified based on available Pk data, consideration of toxicity, and threshold for development of HIV drug resistance. Higher dosing for children who would fall into the lower weight bands (under 3 years) was accepted for drugs with known increased metabolism or clearance in the young child (NVP, 3TC, d4T, abacavir, LPV/r).

In South Africa, the Antiretroviral Drug Dosing Chart (2009) is an update of the 2007 Western Cape version and incorporates elements of the WHO 2008 table; in particular, dosing recommendations for weight bands $3-3.9 \mathrm{~kg}$ and $4-4.9 \mathrm{~kg}$ (Fig. 2). Many of the formulations in the WHO table, in particular the FDCs, are not currently available to public sector treatment programmes in South Africa and so are excluded. The registration and availability of the paediatric strength heat-stable LPV/r tablet (Aluvia; $100 \mathrm{mg}$ lopinavir/25 $\mathrm{mg}$ ritonavir) is still awaited, but it has been included. Local dosing practices described in the 2007 chart have been retained with modification, e.g. the $d 4 \mathrm{~T}$ openedcapsule method is now used from $5 \mathrm{~kg}$ body weight. A comparative analysis of the revised weight band dosing in comparison with BSA dosing for AZT, NVP and L/r has been completed but not yet published. An analysis of the dose of LPV/r solution in $\mathrm{mg} / \mathrm{m}^{2}$ that revised WHO weight band dose recommendations would provide, using anthropometric data on 976 children initiating ART at median age of 11.2 months, indicated doses considerably in excess of $300 / 75 \mathrm{mg} / \mathrm{m}^{2}$, particularly for children $<6$ months of age..$^{19} \mathrm{~A}$ protocol for an infant Pk study using the revised WHO weight-band dosing 


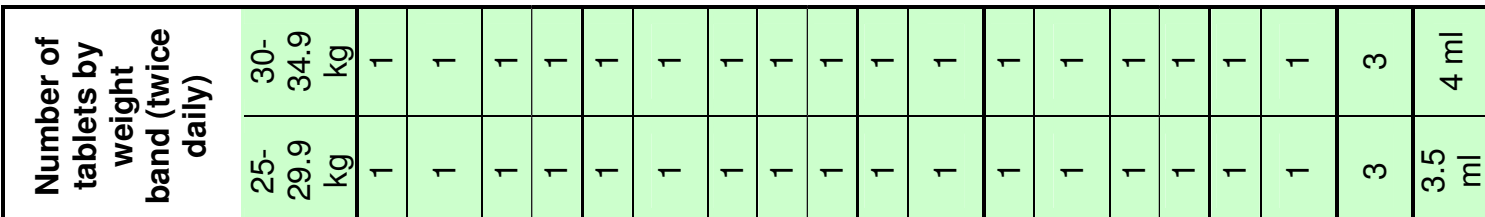
10

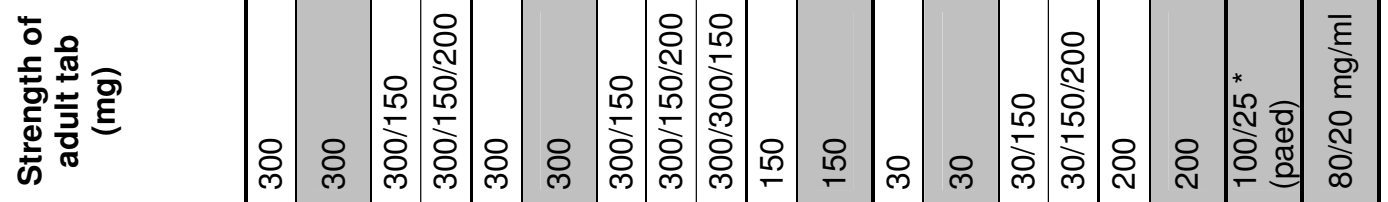

产

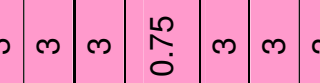

๓

(2)

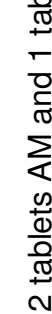

इ)

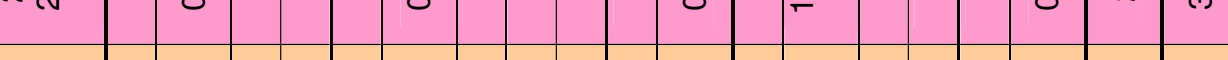

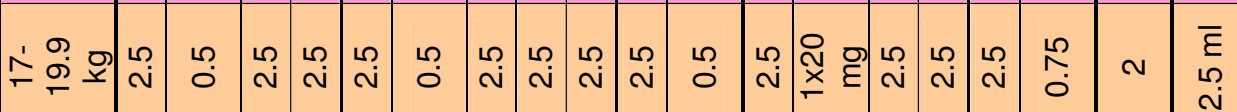

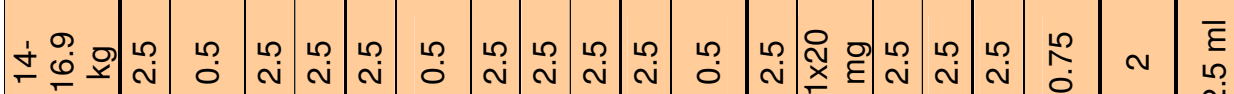

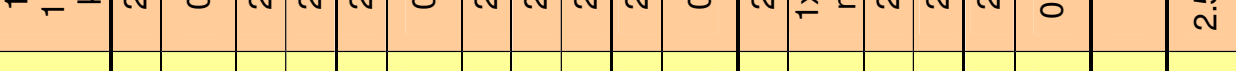

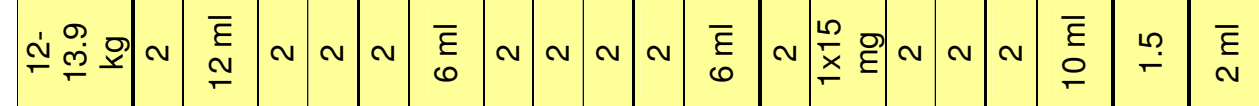

$\stackrel{\frac{2}{2}}{\frac{2}{0}}$

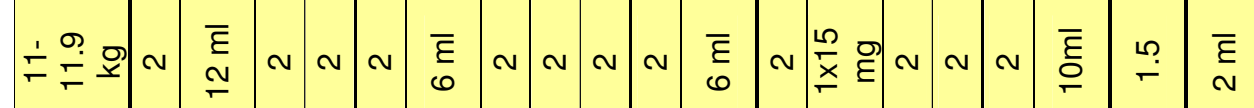

을

은 $\frac{0}{10}$

윰

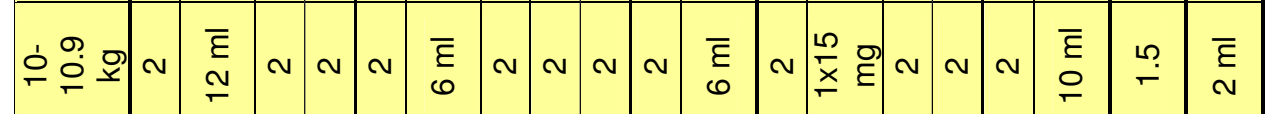

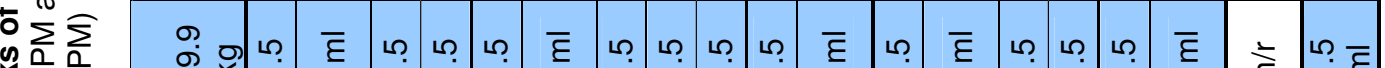

¿ $\frac{\infty}{0}$

$3 \frac{\pi}{0}$

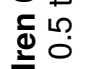

흔 $\frac{0}{0}$

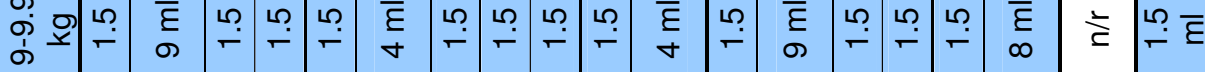

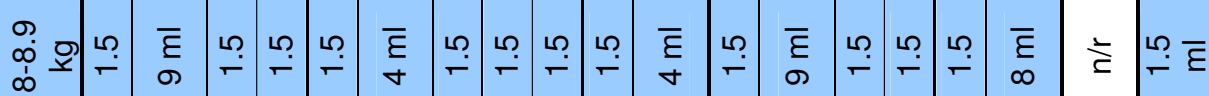

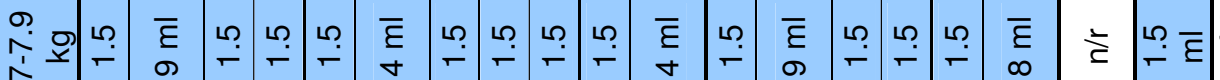

审

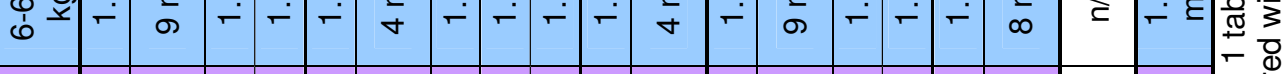

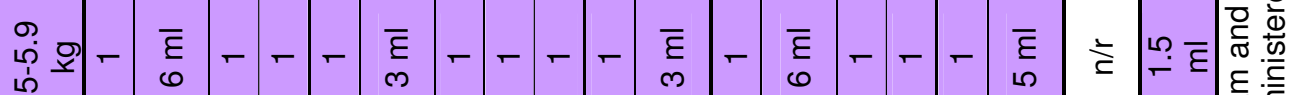

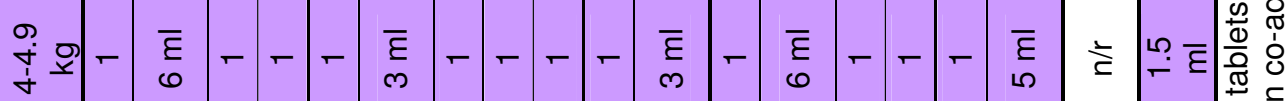

잉

$\stackrel{\frac{1}{\Phi}}{\frac{1}{0}}$

.0

品

$\stackrel{2}{\stackrel{2}{?}}$

o)

仓ํ.

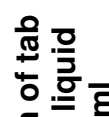

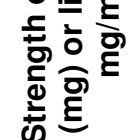

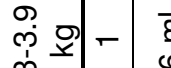

$\bar{\varepsilon}$

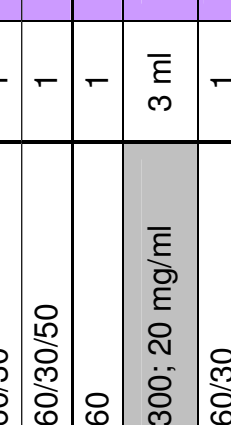

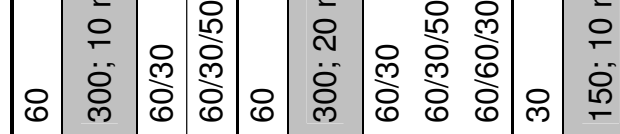

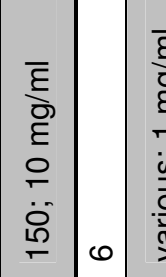

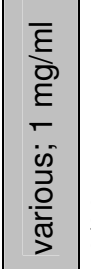

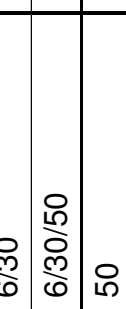

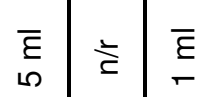

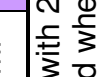
这 

DOSING TABLE (2008) AND ADAPTED FOR THE ANTIRETROVIRAL DRUG DOSING CHART (2009) ${ }^{18}$

\section{Drug Target dosing range \\ $\mathrm{ABC} \quad 8-10 \mathrm{mg} / \mathrm{kg} /$ dose twice daily \\ AZT $\quad 180-240 \mathrm{mg} / \mathrm{m}^{2} /$ dose twice daily}

d4T $\quad 1 \mathrm{mg} / \mathrm{kg} /$ dose twice daily

ddl $\quad<3$ months of age: $50 \mathrm{mg} / \mathrm{m}^{2} /$ dose;

$>3$ months: $120 \mathrm{mg} / \mathrm{m}^{2} /$ dose twice daily

3TC $\quad 4 \mathrm{mg} / \mathrm{kg} /$ dose twice daily

NVP A BSA dose range of $150-200 \mathrm{mg} / \mathrm{m}^{2} /$ dose twice daily is used to generate weight-band dosing

EFV By weight band $(15-18.75 \mathrm{mg} / \mathrm{kg} /$ dose solid formulation or $19.5 \mathrm{mg} / \mathrm{kg} /$ dose suspension, once daily)

LPV/rtv Approved dose is $230 / 75.5 \mathrm{mg} / \mathrm{m}^{2} /$ dose twice daily; $300 / 75 \mathrm{mg} / \mathrm{m}^{2} /$ dose is recommended in children $<2$ years of age, if taken with NNRTI, or for $\mathrm{Pl}$-experienced patients

RTV Co-formulated with lopinavir (L:r ratio 4:1) For patients receiving rifampicin, additional RTV dosed at $0.75 \times \mathrm{L} / \mathrm{r}$ dose to achieve L:r ratio of 1:1
Considerations

Clearance in children $<3$ years old is increased, but recent data on once-daily dosing in children from 3 months of age suggest favourable Pk profile

Twice-daily dosing is acceptable and preferred Dosing at the upper end of the range is recommended for central nervous system HIV disease; dosing at the lower end may be preferred in settings where anaemia is prevalent

Needed as a priority product despite well-recognised longer-term toxicities (lipodystrophy), as it is initially well tolerated, is safer to use in anaemia than AZT, and has lower laboratory monitoring requirements

Avoid over-dosing wherever possible (noting recent revision to adult dosing recommendation to reduce dose) and especially for extended periods to minimise toxicity

Enteric-coated formulations are preferred over the buffered form Needs to be given 1 hour before or 2 hours after food Once-daily dosing accepted over 6 years of age

Clearance in children $<3$ years old is increased, and minimal observed toxicity allows for higher dosing in younger children (up to $5 \mathrm{mg} / \mathrm{kg} /$ dose twice daily)

Under-dosing must be avoided wherever possible owing to low barrier development of HIV drug resistance

A reduced dose (150 - $200 \mathrm{mg} / \mathrm{m}^{2} /$ dose once daily) is recommended for the first 2 weeks when initiating NVP treatment regimens

Young children require a higher NVP dose relative to the NRTI components than delivered in current adult FDCs

Dosing not established for children $<3$ years

Suspension is over $30 \%$ less bio-available than solid formulations

Clearance in children $<2$ years is increased

Actual exposure depends on metabolism and inter-patient variability, which is considerable

Heat-stable paediatric formulation recently approved (awaiting registration in $\mathrm{SA}$ )

Needed for use as a pharmacological booster with PI-based treatment and for children receiving rifampicin-based antituberculosis therapy table and incorporating safety and efficacy end-points has been developed.

\section{CONCLUSION}

The use of weight-band ARV dosing approaches, adapted in accordance with locally available formulations and treatment programme conditions, is a key component of simplifying paediatric ART for health care providers as well as children and their caregivers, and will contribute to expanding treatment coverage for HIV-infected infants and children.

\section{REFERENCES}

1. UNICEF,UNAIDS, WHO and UNFPA. ChildrenandAIDS:ThirdStocktaking Report, 2008. Geneva: UNICEF, 2009. http://www.childinfo.org/files/StocktakingReport08.pdf (accessed 29 September 2009).

2. Violari $A$, Cotton $M$, Gibb $D$, et al. Early antiretroviral therapy and mortality among HIV-infected infants. N Engl J Med 2008; 359: 2233-2244.

3. World Health Organization. Report of the WHO Technical Reference Group, Paediatric HIV/ART Care Guideline Group Meeting WHO Headquarters, Geneva, Switzerland, 10 - 11 April 2008. http://www.who.int/hiv/pub/paediatric/WHO_ Paediatric ART guideline rev mreport 2008.pdf (accessed 8 October 2009).

4. Sohn A, Ananworanich J. How can we simplify antiretroviral therapy in children? Curr Opin HIV AIDS 2007; 2: 426-430.

\section{Acknowledgements}

For assistance with the development, analysis and implementation of the Western Cape Antiretroviral Drug Dosing Chart (2007): Shenaaz Raiman (pharmacist), Heather Jaspan, Brian Eley, Mary-Ann Davies, Brenda Smuts, David Pienaar, Heli Moeng, staff at the HIV clinic at Red Cross War Memorial Children's Hospital, Cape Town and Nolungile HIV clinic, Khayelitsha.

5. Package insert for Abbott Kaletra ${ }^{\circledR}$, Abbott Laboratories, June 2005.

6. Mosteller RD. Simplified calculation of body-surface area. N Engl J Med 1987; 317: 1098.

7. Menson $E$, Walker A, Sharland $M$, et al. Underdosing of antiretrovirals in UK and Irish children with HIV as an example of problems in prescribing medicines to children, 1997-2005: cohort study. BMJ 2006; 332: 1183-1187.

8. Ponnet $\mathrm{M}$, Frederix $\mathrm{K}$, Petdachai $\mathrm{W}$, et al. A drug dosage table is a useful tool to facilitate prescriptions of antiretroviral drugs for children in Thailand. Int J STD AIDS 2005; 16: 420-426.

9. World Health Organisation. Antiretroviral therapy of HIV infection in infants and children in resource-limited settings, towards universal access: Recommendations for a public health approach. 2006. http://www.who.int/hiv/pub/guidelines/en/ (accessed 8 October 2009).

10. O'Brien DP, Sauvageot D, Zachariah R, Humblet P. In resource-limited settings good early outcomes can be achieved in children using adult fixed-dose combination 


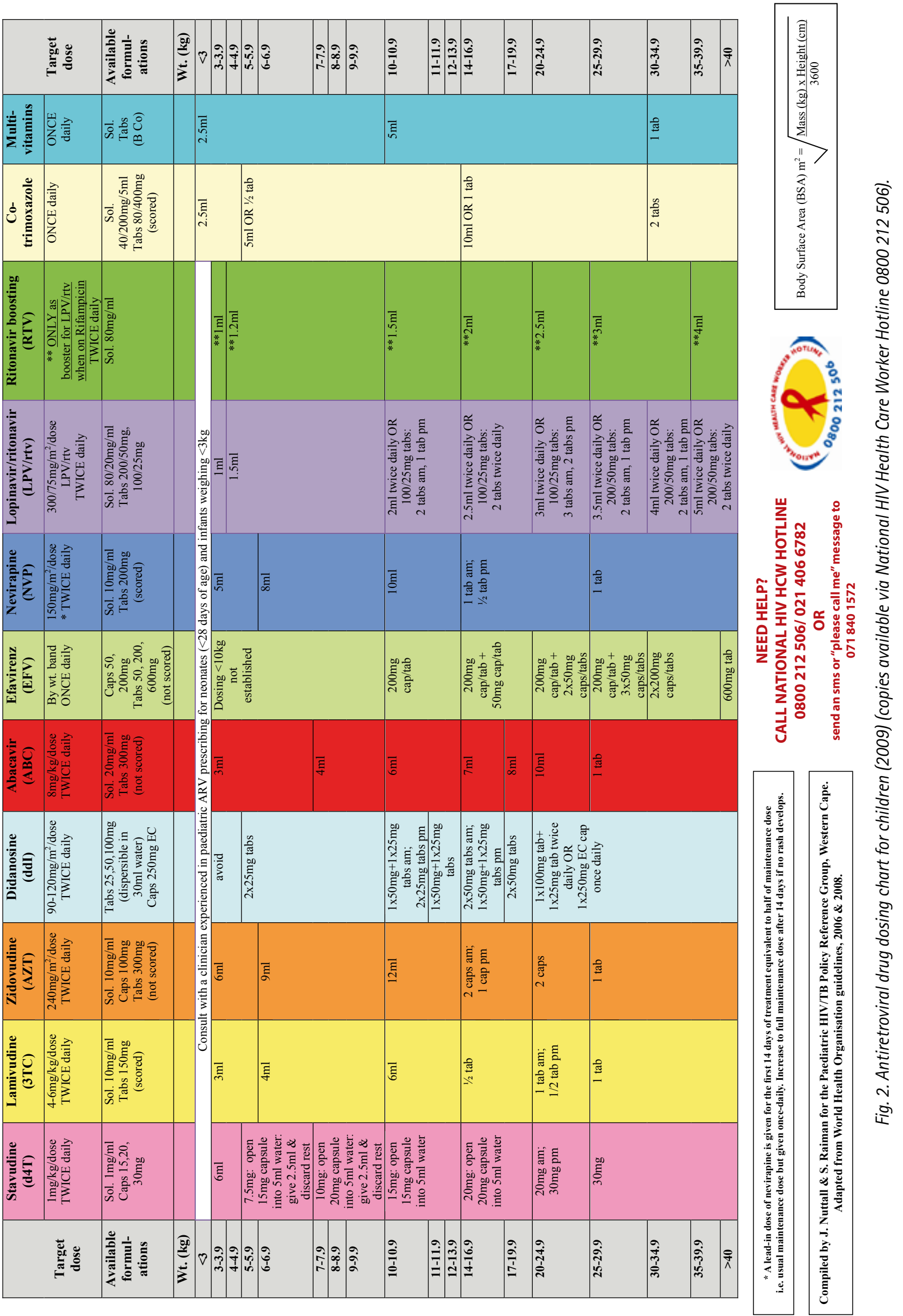


antiretroviral therapy. AIDS 2006; 20: 1955-1960.

11. Callens SF, Westreich $D$, Kitetele $F_{1}$ et al. A visual dosing aid for first-line pediatric antiretroviral treatment in resource-poor settings. J Trop Pediatr 2008; 55: 135137.

12. National Department of Health, South Africa. Guidelines for the Management of HIV-infected Children. 1st ed. National Department of Health, South Africa, 2005.

13. Weidle PJ Abrams EJ Gvetadze R, Rivadeneira E, Kline MW. A simplified weight-based method for pediatric drug dosing for zidovudine and didanosine in resource-limited settings. Pediatr Infect Dis J 2006; 25: 59-64.

14. Innes $S$, Smuts $M$, Cotton $M$, Seifart $H$, Rosenkranz B. Comparative study of different brands of stavudine capsules for the off-label 'opened capsule' method recommended for HIV-infected infants and children in resource-limited settings. South African Journal of Child Health 2009; 3: 44-47.

15. Ren $Y$, Nuttall J, Egbers $C$, et al. Effect of rifampicin on lopinavir pharmacokinetics in HIV-infected children with tuberculosis. J Acquir Immune Defic Syndr 2008: 47: 566-569
16. Nuttall J, Eley B, Davies M. Comparison of body surface area-based dosing and a simplified weight-based dosing method for zidovudine, didanosine, nevirapine and lopinavir/ritonavir in children starting antiretroviral therapy. Paper presented at the 3rd South African AIDS Conference, 5 - 8 June 2007, Durban (Abstract 538).

17. World Health Organization. Annex E: Simplified weight based Paediatric dosing for antiretroviral medicines http://www.who.int/hiv/topics/paediatric/technical/en/ index.html (accessed 17 November 2009).

18. World Health Organization. Report of the WHO expert working group meeting to determine preferred ARV medicines for treating and preventing HIV infection in

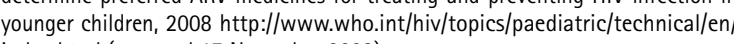
index.html (accessed 17 November 2009).

19. Nuttall J, Eley B, Davies M-A. How well do the revised World Health Organization weight-based dosing guidelines for lopinavir/ritonavir in infants and children correlate with body surface area-based dosing recommendations? Paper presented at the 5th International AIDS Society Conference on HIV Pathogenesis, Treatment and Prevention, 19 - 22 July 2009, Cape Town (Abstract MOAB105). 